Article

\title{
New Similarity Solutions of a Generalized Variable-Coefficient Gardner Equation with Forcing Term
}

\author{
Jianping Zhou ${ }^{1}$, Yuan Wang ${ }^{1}$, Yang Wang ${ }^{1}$, Zhilian Yan ${ }^{2, *}$ and Zhen Wang ${ }^{3}$ (]) \\ 1 School of Computer Science \& Technology, Anhui University of Technology, Ma'anshan 243032, China; \\ jpzhou0@gmail.com (J.Z.); yuanwang0719@gmail.com (Yu.W.); yang91wang@gmail.com (Ya.W.) \\ 2 School of Mathematics \& Physics, Anhui University of Technology, Ma'anshan 243032, China \\ 3 College of Mathematics \& Systems Science, Shandong University of Science \& Technology, \\ Qingdao 266590, China; wangzhen.sd@gmail.com \\ * Correspondence: zhilianyan@gmail.com; Tel.: +86-139-5556-9350
}

Received: 2 April 2018; Accepted: 12 April 2018; Published: 16 April 2018

\begin{abstract}
The compatibility method is used for a generalized variable-coefficient Gardner equation (GVGE) with a forcing term. By the compatibility of the considered equation and a non-classical symmetry of a given form, four types of symmetry are obtained. Then, by solving the characteristic equations of symmetry, the GVGE is reduced to variable coefficients ordinary differential equations, and rich varieties of new similarity solutions are presented. Our results show that the compatibility method can be employed for variable coefficients nonlinear evolution equations with forcing terms.
\end{abstract}

Keywords: Gardner equation; compatibility method; symmetry; similarity solution

\section{Introduction}

In the area of mathematics and physics, a considerable number of systems, ranging from gravitational dynamics and plasma dynamics to thermodynamics, can be modeled by nonlinear evolution equations. Exact solutions of a nonlinear evolution equation offer direct and valuable insight into the physical aspects of the problem modeled by the equation. Therefore, the search for exact solutions of nonlinear evolution equations has gained much attention in the past decades from the mathematical physics community, and a large number of methods have been proposed, such as the tanh method [1,2], the inverse scattering method [3], the homogeneous balance method [4], the $\left(G^{\prime} / G\right)$-expansion method [5,6], the sine-cosine method [7], the Frobenius integrable decomposition method [8], the improved Exp-function method [9], the generalized Kudryashov method [10], the local fractional Riccati differential equation method [11], the Hirota bilinear method [12-14], the Darboux transformation method [15,16] and the group methods [17-25]. As stated in [26], symmetry is the key to solving differential equations. In 2006, a systematic method (named the compatibility method) was developed in [27] to seek non-classical symmetries and similarity solutions of a class of variable coefficients Zakharov-Kuznetsov equations. After that, the method was extended to investigate the high-dimensional breaking soliton equation [28], the variable coefficients Broer-Kaup system [29], the Wick-type stochastic Korteweg-de Vries equation [30] and the variable coefficients coupled KdVsystem [31], consecutively. As shown in [27-31], the method is capable of obtaining abundant symmetry reductions and similarity solutions of the considered nonlinear evolution equations. In addition, it is able to greatly reduce the computational complexity in comparison to the non-classical group methods (see, for instance, $[17,18]$ ). 
In this paper, the compatibility method will be used to solve the generalized variable coefficients Gardner equation (GVGE) with the forcing term given in [32]:

$$
u_{s}+a(s) u u_{x}+b(s) u^{2} u_{x}+h(s) u_{x x x}+d(s) u_{x}+f(s) u=R(s),
$$

where $a(s), b(s), h(s) \neq 0, d(s), f(s)$ and $R(s)$ are arbitrary smooth functions of $s$. It is worth pointing out that Equation (1) is widely applied in different physical areas, including nonlinear lattice, hydrodynamics, plasma physics, fluid dynamics and quantum field theory. Let the forcing term $R(s)=0$; Equation (1) turns into:

$$
u_{s}+a(s) u u_{x}+b(s) u^{2} u_{x}+h(s) u_{x x x}+d(s) u_{x}+f(s) u=0 .
$$

In [33], with symbolic computation, the Bäcklund transformation, Lax pair and N-soliton-like solution of Equation (2) were investigated. By employing the Hirota bilinear method, a number of multi-solitary-wave solutions of Equation (2) was proposed in [34]. By using the sine-Gordon expansion method, new solitary and optical wave structures solutions of Equation (2) were given in [35]. In addition, let $b(s)=0$; Equation (1) changes into the variable coefficient $\mathrm{KdV}$ equation:

$$
u_{s}+a(s) u u_{x}+h(s) u_{x x x}+d(s) u_{x}+f(s) u=R(s) .
$$

In [36], the extended mapping method was used to obtain new exact solutions of Equation (3), including soliton solutions, periodic solutions and rational solutions.

The rest of the paper is outlined as follows. In Section 2, by using the compatibility method, we obtain a series of symmetries of Equation (1). In Section 3, we give the symmetry reductions of Equation (1). In Section 4, we derive some similarity solutions of Equation (1). In the last section, we present the conclusions.

\section{Symmetry}

The fundamental idea of the compatibility method is to exploit a non-classical symmetry of a given nonlinear evolution equation such as Equation (1) as follows:

$$
u_{s}=\alpha(x, s) u_{x}-\Phi(x, s, u(x, s)) .
$$

In Equation (4), $\alpha(x, s)$ and $\Phi(x, s, u(x, s))$ are functions to be determined later by the compatibility of Equations (1) and (4). By substituting Equation (4) into Equation (1), we have:

$$
\begin{aligned}
u_{x x x}= & \left(R(s)-\alpha(x, s) u_{x}+\Phi(x, s, u(x, s))-a(s) u u_{x}\right. \\
& \left.\left.-b(s) u^{2} u_{x}-d(s) u_{x}-f(s) u\right)\right) / h(s) .
\end{aligned}
$$

In terms of the equality in $u_{s s}$ of Equations (1) and (4), we can obtain:

$$
\begin{aligned}
& \left(R(s)-\alpha(s) u u_{x}-b(u) u^{2} u_{x}-h(s) u_{x x x}-d(s) u_{x}-f(s) u\right)_{s} \\
= & \left(\alpha(x, s) u_{x}-\Phi(x, s, u(x, s))\right)_{s} .
\end{aligned}
$$

Expanding Equation (6) and substituting Equations (4) and (5) into it result in the following equation with the help of Maple:

$$
3 h(s) \Phi_{x u u} u_{x}^{2}+h(s) \Phi_{u u u} u_{x}^{3}+F_{1}\left(x, s, u, u_{x}, \ldots\right)=0,
$$

where $F_{1}$ is not dependent on $u_{x}^{2}$ and $u_{x}^{3}$. In order to guarantee Equation (7) for an arbitrary solution $u$, we set the coefficients of $u_{x}^{2}$ and $u_{x}^{3}$ to be zero. Then, we obtain: 


$$
\Phi_{x u u}=\Phi_{u u u}=0 \text {, i.e., } \Phi(x, s, u(x, s))=\beta(s) u^{2}+\gamma(x, s) u+\delta(x, s) .
$$

In Equation (8), $\beta(s), \gamma(x, s)$ and $\delta(x, s)$ are functions to be solved later. Based on Equation (8), Equation (7) can be simplified as:

$$
-2 b(s) \beta(s) h(s) u^{3} u_{x}-b(s) \gamma_{x} h(s) u^{3}+F_{2}\left(x, s, u, u_{x}, \ldots\right)=0,
$$

where $F_{2}$ is not dependent on $u^{3} u_{x}$ and $u^{3}$, which means $\beta(s)=\gamma_{x}=0$. Following the same steps, we can obtain:

$$
\alpha(x, s)=\alpha_{1}(s) x+\alpha_{2}(s), \Phi(x, s, u(x, s))=\gamma(s) u+\delta(s),
$$

where $\alpha_{1}(s), \alpha_{2}(s), \gamma(s)$ and $\delta(s)$ are functions to be determined. The determining equations are as follows:

$$
\begin{aligned}
& h^{\prime}(s) \alpha_{1}(s)-h(s) \alpha_{1}^{\prime}(s)+3 \alpha_{1}^{2}(s) h(s)=0, \\
& h^{\prime}(s) f(s)+h(s) \gamma^{\prime}(s)-f^{\prime}(s) h(s)-h^{\prime}(s) \gamma(s)-3 \alpha_{1}(s) h(s) \gamma(s)+3 \alpha_{1}(s) h(s) f(s)=0, \\
& h^{\prime}(s) \alpha_{2}(s)-h(s) \alpha_{2}^{\prime}(s)-d^{\prime}(s) h(s)+h^{\prime}(s) d(s)+3 \alpha_{1}(s) \alpha_{2}(s) h(s) \\
+ & 2 \alpha_{1}(s) h(s) d(s)+\alpha(s) \delta(s) h(s)=0, \\
& -h^{\prime}(s) R(s)+h(s) R^{\prime}(s)+\delta^{\prime}(s) h(s)-h^{\prime}(s) \delta(s)-3 \alpha_{1}(s) \delta(s) h(s) \\
- & 3 \alpha_{1}(s) h(s) R(s)+f(s) \delta(s) h(s)+\gamma(s) h(s) R(s)=0, \\
& h^{\prime}(s) b(s)-h(s) b^{\prime}(s)+2 \alpha_{1}(s) h(s) b(s)+2 b(s) h(s) \gamma(s)=0, \\
& h^{\prime}(s) a(s)-h(s) a^{\prime}(s)+2 \alpha_{1}(s) h(s) a(s)+2 b(s) h(s) \delta(s)+a(s) \gamma(s) h(s)=0 .
\end{aligned}
$$

According to $\alpha_{1}(s), a(s)$ and $b(s)$, we discuss the following four concrete cases: $\left(\mathbf{i}_{1}\right) \alpha_{1}(s) \neq 0, a(s) \neq 0$ and $b(s) \neq 0$

First consider the Equation (10), which is a Bernoulli equation of $\alpha_{1}(s)$. Solving Equation (10), we have:

$$
\alpha_{1}(s)=h(s) /\left(\int-3 h(s) d s+C_{1}\right),
$$

where $C_{1}$ is an integral constant. Solving Equations (11)-(13) leads to:

$$
\begin{aligned}
\gamma(s) & =f(s)-C_{2} \alpha_{1}(s), \\
\delta(s) & \left.=\alpha_{1}(s) \exp \left(-\int f(s) d s\right)\left(\int \Delta_{1}(s) \exp \left(\int f(s) d s\right) / \alpha_{1}(s) h(s)\right) d s+C_{3}\right), \\
\alpha_{2}(s) & =\alpha_{1}(s)\left(\int\left(\Delta_{2}(s) / \alpha_{1}(s) h(s)\right)\right) d s+C_{4},
\end{aligned}
$$

where $C_{2}, C_{3}$ and $C_{4}$ are integral constants,

$$
\begin{aligned}
& \Delta_{1}(s)=h^{\prime}(s) R(s)-h(s) R^{\prime}(s)+3 \alpha_{1}(s) h(s) R(s)-R(s) h(s) f(s)+C_{2} h(s) R(s) \alpha_{1}(s), \\
& \Delta_{2}(s)=h^{\prime}(s) d(s)-h(s) d^{\prime}(s)+2 \alpha_{1}(s) h(s) d(s)+a(s) \delta(s) h(s) .
\end{aligned}
$$

In addition, $a(s), b(s), h(s), d(s), f(s)$ and $R(s)$ satisfy certain constraint conditions:

$$
\begin{gathered}
2 b(s) h(s)\left(\alpha_{1}(s)+f(s)-C_{2} \alpha_{1}(s)\right)+b(s) h^{\prime}(s)-h(s) b^{\prime}(s)=0, \\
a(s) h(s)\left(2 \alpha_{1}(s)+f(s)-C_{2} \alpha(s)\right)+a(s) h^{\prime}(s)-h(s) a^{\prime}(s)+2 b(s) \delta(s) h(s)=0 .
\end{gathered}
$$

Thus, Equation (1) allows symmetry as follows: 


$$
\sigma_{1}=\left(\alpha_{1}(s) x+\alpha_{2}(s)\right) u_{x}-u_{s}-\gamma(s) u-\delta(s),
$$

where $\alpha_{1}(s), \alpha_{2}(s), \gamma(s)$ and $\delta(s)$ are given in Equations (16) and (17).

$\left(\mathbf{i}_{2}\right) \alpha_{1}(s) \neq 0, a(s) \neq 0$ and $b(s)=0$

In this case, Equation (1) turns into Equation (3). Solving Equations (11)-(13), we obtain:

$$
\begin{gathered}
\alpha_{1}(s)=h(s) /\left(\int-3 h(s) d s+C_{1}\right), \\
\gamma(s)=f(s)-C_{2} \alpha_{1}(s), \\
\alpha_{2}(s)=\alpha_{1}(s)\left(\int\left(\Delta_{2}(s) / \alpha_{1}(s) h(s)\right) d s+C_{4}\right), \\
\left.\left.\delta(s)=\alpha_{1}(s) \exp \left(-\int f(s) d s\right)\right)\left(\int\left(\Delta_{1}(s) \exp \left(\int f(s) d s\right)\right) / \alpha_{1}(s) h(s)\right) d s+C_{3}\right),
\end{gathered}
$$

where $C_{1}, C_{2}, C_{3}$ and $C_{4}$ are integral constants,

$$
\Delta_{1}(s)=h^{\prime}(s) R(s)-h(s) R^{\prime}(s)+3 \alpha_{1}(s) h(s) R(s)-R(s) h(s) f(s)+C_{2} h(s) R(s) \alpha_{1}(s),
$$

and:

$$
\Delta_{2}(s)=h^{\prime}(s) d(s)-h(s) d^{\prime}(s)+2 \alpha_{1}(s) h(s) d(s)+a(s) \delta(s) h(s),
$$

where $a(s), b(s), h(s), d(s), f(s)$ and $R(s)$ satisfy the constraint condition:

$$
a(s) h(s)\left(2 \alpha_{1}(s)+f(s)-C_{2} \alpha_{1}(s)\right)+a(s) h^{\prime}(s)-h(s) a^{\prime}(s)=0 .
$$

Thus, Equation (3) admits the symmetry:

$$
\sigma_{2}=\left(\alpha_{1}(s) x+\alpha_{2}(s)\right) u_{x}-u_{s}-\gamma(s) u-\delta(s),
$$

where $\alpha_{1}(s), \alpha_{2}(s), \gamma(s)$ and $\delta(s)$ are given by Equation (21).

Remark 1. Let $a(s)=1, h(s)=b_{0} e^{(m-2) a_{0} s}, d(s)=R(s)=0, f(s)=a_{0}, C_{1}=C_{3}=C_{4}=0$, $C_{2}=3 /(2-m)-1$, then we have $\sigma_{2}=\alpha_{0}(m-2) x u_{x}+3 u_{s}-a_{0}(m-2) u$, which is the symmetry generator $X_{3}$ in [37] of $a(s)=a_{0}, b(s)=b_{0} e^{(m-2) a_{0} s}$. Let $a(s)=1, b(s)=b_{0} s^{m}, d(s)=R(s)=0, f(s)=1 / s$, $C_{1}=C_{3}=C_{4}=0, C_{2}=-1$, then we obtain $\sigma_{2}=(m+1) x u_{x}+3 t u_{s}-(m-2) u$, which is the symmetry generator $X_{3}$ in [37] of $a(s)=1 / s, b(s)=b_{0} s^{m}$.

$\left(\mathbf{i}_{3}\right) \alpha_{1}(s) \neq 0, a(s)=0$ and $b(s) \neq 0$

In this case, Equation (1) becomes the variable coefficient mKdVequation, which allows symmetry as follows:

$$
\sigma_{3}=\left(\alpha_{1}(s) x+\alpha_{2}(s)\right) u_{x}-u_{s}-\gamma(s) u,
$$

where:

$$
\begin{aligned}
\alpha_{1}(s) & =h(s) /\left(\int-3 h(s) d s+C_{1}\right), \\
\gamma(s) & =f(s)-C_{2} \alpha_{1}(s), \alpha_{2}(s)=\alpha_{1}(s)\left(\int\left(\Delta_{2}(s) / \alpha_{1}(s) h(s)\right) d s+C_{4}\right),
\end{aligned}
$$

$C_{1}, C_{2}$ and $C_{4}$ are integral constants, and:

$$
\Delta_{2}(s)=h^{\prime}(s) d(s)-h(s) d^{\prime}(s)+2 \alpha_{1} h(s) d(s),
$$


$b(s), h(s), d(s), f(s)$ and $R(s)$ satisfy certain constraint conditions:

$$
\begin{gathered}
2 b(s) h(s)\left(\alpha_{1}(s)+f(s)-C_{2} \alpha_{1}(s)\right)+b(s) h^{\prime}(s)-h(s) b^{\prime}(s)=0, \\
h(s) R(s)\left(f(s)-3 \alpha_{1}(s)-C_{2} \alpha_{1}(s)\right)+R(s) h^{\prime}(s)+h(s) R^{\prime}(s)=0 .
\end{gathered}
$$

Remark 2. Let $b(s)=1, h(s)=b_{0}, d(s)=R(s)=f(s)=0, C_{1}=C_{4}=0, C_{2}=1$, then we have $\sigma_{3}=x u_{x}+3 t u_{s}+u$, which is the symmetry generator $X_{3}$ in [38] of $a(s)=0, b(s)=b_{0}$. Let $b(s)=1, h(s)=$ $b_{0} e^{m s}, d(s)=R(s)=f(s)=0, C_{1}=C_{4}=0, C_{2}=-1 / 2$, then it leads to $\sigma_{3}=m x_{x} u / 3+u_{s}-m u / 6$, which is the symmetry generator $X_{3}$ in [38] of $a(s)=0, b(s)=b_{0} s^{m}$. Thus, we generalize the results in [38].

$\left(\mathbf{i}_{4}\right) \alpha_{1}(s)=0, a(s)$ and $b(s)$ are arbitrary functions

In this case, we have:

$$
\begin{aligned}
\gamma(s)= & f(s)-C_{1} h(s), \\
\alpha_{2}(s)= & h(s)\left(C_{2}+\int(\alpha(s) \delta(s) / h(s)) d s\right)-d(s), \\
\delta(s)= & \left.\exp \left(\int(-f(s) d s)+h^{\prime}(s) / h(s)\right) d s\right) \\
& \times\left(C_{3}+\int \epsilon(s)\left(R(s) h^{\prime}(s) / h(s)-R^{\prime}(s)-R(s) \gamma(s)\right) d s\right),
\end{aligned}
$$

where $\epsilon(s)=\exp \left(\int\left(f(s)-h^{\prime}(s) / h(s)\right) d s\right), C_{1}, C_{2}$ and $C_{3}$ are integral constants, In addition, $a(s), b(s), h(s), d(s), f(s)$ and $R(s)$ satisfy the constraint condition:

$$
\begin{gathered}
2 b(s) h(s) \gamma(s)+b(s) h^{\prime}(s)-h(s) b^{\prime}(s)=0, \\
a(s) h(s) \gamma(s)+a(s) h^{\prime}(s)-h(s) a^{\prime}(s)+2 b(s) \delta(s) h(s)=0,
\end{gathered}
$$

Thus, Equation (1) admits the symmetry:

$$
\sigma_{4}=\alpha_{2}(s) u_{x}-u_{s}-\gamma(s) u-\delta(s),
$$

where $\alpha_{2}(s), \gamma(s)$ and $\delta(s)$ are determined by Equation (27).

\section{Symmetry Reduction}

In order to obtain the symmetry reduction of the considered equation by the compatibility method, we should first solve the associated characteristic equations of $\sigma=0$ to get similarity variables and then substitute them into Equation (1) to obtain the corresponding reduced equations.

$\left(\mathbf{j}_{1}\right) \sigma_{1}=\left(\alpha_{1}(s) x+\alpha_{2}(s)\right) u_{x}-u_{s}-\gamma(s) u-\delta(s)$

The characteristic equations of $\sigma_{1}=0$ are as follows:

$$
\frac{d x}{\alpha_{1}(s) x+\alpha_{2}(s)}=\frac{d s}{-1}=\frac{d u}{\gamma(s) u+\delta(s)},
$$

where $\alpha_{1}(s), \alpha_{2}(s), \gamma(s)$ and $\delta(s)$ are given in Equations (16) and (17). Solving Equation (31), we can obtain the following expression:

$$
u=\exp \left(-\int \gamma(s) d s\right)\left(F(\xi)-\int \delta(s) \exp \left(\int \gamma(s) d s\right) d s\right),
$$

where: 


$$
\left.\xi=x \exp \left(\int \alpha_{1}(s) d s\right)+\int \alpha_{2}(s) \exp \left(\int \alpha_{1}(s) d s\right) d s\right)
$$

and $F$ are similarity variables. By substituting Equation (32) into Equation (1), we have a reduced equation in the form:

$$
F^{\prime \prime \prime}+c_{1} F^{2} F^{\prime}+c_{2} F+c_{3} F^{\prime} F+c_{4} \xi F^{\prime}+c_{5} F^{\prime}+c_{6}=0
$$

which is a third-order variable coefficients ordinary differential equation of $F(\xi)$, where $c_{1}, c_{2}, c_{3}, c_{4}$, $c_{5}$ and $c_{6}$ are coefficients, and:

$$
\begin{aligned}
c_{1}= & b(s) \exp \left(-2 \int\left(\gamma(s)+\alpha_{1}(s)\right) d s\right) / h(s), \\
c_{2}= & c_{2} \alpha_{1}(s) \exp \left(-3 \int \alpha_{1}(s) d s\right) / h(s), \\
c_{3}= & \alpha(s) \exp \left(-\int\left(\gamma(s)+2 \alpha_{1}(s)\right) d s\right) / h(s)-2 c_{1} \int \delta(s) \exp \left(\int \gamma(s) d s\right) d s, \\
c_{4}= & c_{2} / C_{2}, \\
c_{5}= & \left(\alpha_{2}(s)+d(s)-\alpha(s) \exp \left(-\int \gamma(s) d s\right) \int \delta(s) \exp \left(\int \gamma(s) d s\right) d s\right) \\
& \times \exp \left(-2 \int \alpha_{1}(s) d s\right) / h(s)+c_{1}\left(\int \delta(s) \exp \left(\int \gamma(s) d s\right) d s\right)^{2} \\
& -\alpha_{1}(s) \exp \left(-3 \int \alpha_{1}(s) d s\right) \int \alpha_{2}(s) \exp \left(\int \alpha_{1}(s) d s\right) d s / h(s), \\
c_{6}= & \left.-(R(s)+\delta(s)) \exp \left(\int \gamma(s)-3 \alpha_{1}(s)\right) d s\right) / h(s)-c_{2} \int \delta(s) \exp \left(\int \gamma(s) d s\right) d s .
\end{aligned}
$$

Note that when $c_{2}=c_{4}$, integrating Equation (33) once about $\xi$ yields:

$$
F^{\prime \prime}+c_{1} F^{3} / 3+c_{2} \xi F+c_{3} F^{2} / 2+c_{5} F+c_{6} \xi+c_{7}=0,
$$

where $c_{7}$ is an integral constant. If $c_{1}=c_{2}=c_{5}=c_{7}=0, c_{3}=-12, c_{6}=-1$, Equation (34) is the Painleve I equation. If $c_{3}=c_{5}=c_{6}=0, c_{1}=-6, c_{2}=-1$, Equation (34) is the Painleve II equation. If $c_{2}=c_{6}=0$, Equation (34) is the elliptic equation.

$\left(\mathbf{j}_{2}\right) \sigma_{2}=\left(\alpha_{1}(s) x+\alpha_{2}(s)\right) u_{x}-u_{s}-\gamma(s) u-\delta(s)$

The characteristic equations of $\sigma_{2}=0$ are as follows:

$$
\frac{d x}{\alpha_{1}(s) x+\alpha_{2}(s)}=\frac{d s}{-1}=\frac{d u}{\gamma(s) u+\delta(s)},
$$

where $\alpha_{1}(s), \alpha_{2}(s), \gamma(s)$ and $\delta(s)$ are given in Equation (21). Solving the above equations, we get:

$$
u=\exp \left(-\int \gamma(s) d s\right)\left(F(\xi)-\int \delta(s) \exp \left(\int \gamma(s) d s\right) d s\right)
$$

where:

$$
\xi=x \exp \left(\int \alpha_{1}(s) d s\right)+\int \alpha_{2}(s) \exp \left(\int \alpha_{1}(s) d s\right) d s,
$$

and $F$ are similarity variables. By substituting the expression into Equation (3), we have a reduced equation in the form:

$$
F^{\prime \prime \prime}+c_{1} F+c_{2} F^{\prime} F+c_{3} \xi F^{\prime}+c_{4} F^{\prime}+c_{5}=0,
$$

where $c_{1}, c_{2}, c_{3}, c_{4}$ and $c_{5}$ are coefficients, which are similar to the coefficients of Equation (33), except for $b(s)=0$.

$\left(\mathbf{j}_{3}\right) \sigma_{3}=\left(\alpha_{1}(s) x+\alpha_{2}(s)\right) u_{x}-u_{s}-\gamma(s) u$ 
The characteristic equations of $\sigma_{3}=0$ are as follows:

$$
\frac{d x}{\alpha_{1}(s) x+\alpha_{2}(s)}=\frac{d s}{-1}=\frac{d u}{\gamma(s) u}
$$

where $\alpha_{1}(s), \alpha_{2}(s)$ and $\gamma(s)$ are given in Equation (24). Solving the above system, we get:

$$
u=F(\xi) \exp \left(-\int \gamma(s) d s\right)
$$

where:

$$
\xi=x \exp \left(\int \alpha_{1}(s) d s\right)+\int \alpha_{2}(s) \exp \left(\int \alpha_{1}(s) d s\right) d s,
$$

and $F$ are similarity variables. By substituting the expression into Equation (1) with $\alpha(s)=0$, we have a reduced equation in the form:

$$
F^{\prime \prime \prime}+c_{1} F+c_{2} F^{\prime} F^{2}+c_{3} \xi F^{\prime}+c_{4} F^{\prime}+c_{5}=0,
$$

where $c_{1}, c_{2}, c_{3}, c_{4}$ and $c_{5}$ are coefficients, which are similar to the coefficients of Equation (33) except for $\alpha(s)=0$ and $\xi(s)=0$.

$\left(\mathbf{j}_{4}\right) \sigma_{4}=\alpha_{2}(s) u_{x}-u_{s}-\gamma(s) u-\delta(s)$

The characteristic equations of $\sigma_{4}=0$ are as follows:

$$
\frac{d x}{\alpha_{2}(s)}=\frac{d s}{-1}=\frac{d u}{\gamma(s) u+\delta(s)}
$$

where $\alpha_{2}(s), \gamma(s)$ and $\delta(s)$ are given by Equations (28) and (29). Solving the above equations, we get:

$$
u=\exp \left(-\int \gamma(s) d s\right)\left(F(\xi)-\int \delta(s) \exp \int(\gamma(s) d s) d s\right),
$$

where:

$$
\xi=x+\int \alpha_{2}(s) d s
$$

and $F$ are similarity variables. By substituting the expression into Equation (1), we have a reduced equation in the form:

$$
F^{\prime \prime \prime}+c_{1} F^{2} F^{\prime}+c_{2} F+c_{3} F F^{\prime}+c_{4} F^{\prime}+c_{5}=0,
$$

where $c_{1}, c_{2}, c_{3}, c_{4}$ and $c_{5}$ are coefficients given by:

$$
\begin{aligned}
c_{1}= & b(s) \exp \left(-2 \int \gamma(s) d s\right) / h(s), c_{2}=c_{1}, \\
c_{3}= & a(s) \exp \left(-\int \gamma(s) d s\right) / h(s)-2 b(s) \exp \left(-2 \int \gamma(s) d s\right)\left(\int \delta(s)\right. \\
& \left.\times \exp \left(\int \gamma(s) d s\right) d s\right) / h(s), \\
c_{4}= & \left(\alpha_{2}(s)+d(s)\right) / h(s)-a(s) \exp \left(-\int \gamma(s) d s\right)\left(\int \delta(s) \exp \left(\int \gamma(s) d s\right) d s\right) / h(s) \\
& \left.+b(s) \exp \left(-2 \int \gamma(s) d s\right)\left(\int \delta(s) \exp \left(\int \gamma(s) d s\right) d s\right)^{2} / h(s)\right), \\
c_{5}= & -(R(s)+\delta(s)) \exp \left(\int \gamma(s) d s\right) / h(s)-c_{2} \int \delta(s) \exp \left(\int \gamma(s) d s\right) d s .
\end{aligned}
$$




\section{Similarity Solutions}

In this section, we can derive the corresponding similarity solutions of the GVGE by solving the obtained reduced equation in Section 3. Solving Equation (33) or Equation (34) leads to the following solutions of $F(\xi)$ :

$$
\begin{aligned}
F_{1}(\xi) & =D_{1} \exp (-\xi)+\exp (\xi / 2)\left(D_{2} \sin (\sqrt{3} \xi / 2)+D_{3} \cos (\sqrt{3} \xi / 2)\right) \\
c_{1} & =c_{3}=c_{4}=c_{5}=c_{6}=0, c_{2}=1 \\
F_{2}(\xi) & =D_{1}+D_{2}+\int A i(-\xi) d \xi+D_{3} \int B i(-\xi) d \xi \\
c_{1} & =c_{2}=c_{3}=c_{5}=c_{6}=0, c_{4}=1 \\
F_{3}(\xi) & =D_{1} \xi^{2}+D_{2} \xi+D_{3}, c_{1}=c_{2}=c_{3}=c_{4}=c_{5}=c_{6}=0 \\
F_{4}(\xi) & =D_{1}+D_{2} \exp (-k \xi)+D_{3} \exp (k \xi), c_{1}=c_{2}=c_{3}=c_{4}=c_{6}=0 \\
c_{5} & =-k^{2} \\
F_{5}(\xi) & =D_{1}+D_{2} \sin (k \xi)+D_{3} \cos (k \xi), c_{1}=c_{2}=c_{3}=c_{4}=c_{6}=0 \\
c_{5} & =k^{2} \\
F_{6}(\xi) & = \pm \tanh (\xi), \pm \operatorname{coth}(\xi), c_{1}=-6, c_{5}=2, c_{2}=c_{3}=c_{6}=c_{7}=0 \\
F_{7}(\xi) & =6 \sec h(\xi) /(\sqrt{13}+\sec h(\xi)), c_{1}=2, c_{3}=1, c_{5}=-1 \\
c_{2} & =c_{6}=c_{7}=0, \\
F_{8}(\xi) & = \pm \tan (\xi), \pm \cot (\xi), c_{1}=-6, c_{5}=-2, c_{2}=c_{3}=c_{6}=c_{7}=0 \\
F_{9}(\xi) & =D_{1}+3 D_{1} \tan \left(\sqrt{3 D_{1} / 2}\left(\xi+D_{2}\right)\right) / 2, c_{1}=c_{2}=c_{5}=c_{6}=0 \\
c_{3} & =-12, c_{7}=3 D_{1}^{2} / 2\left(D_{1}>0\right) \\
F_{10}(\xi) & =1 /\left(1+D_{1} \xi+D_{2} \xi^{2}\right), c_{2}=c_{4}=c_{5}=c_{6}=0, c_{1}=-6\left(D_{1}^{2}-4 D_{2}\right) \\
c_{3} & =-12 D_{2} \\
F_{11}(\xi) & =1 /\left(1+c n^{2} \xi\right), c_{2}=c_{5}=c_{6}=0, c_{1}=144 / 5, c_{3}=-84 / 5, c_{7}=2 / 5 \\
F_{12}(\xi) & =\sqrt{2} c n \xi /(\operatorname{sn} \xi+\sqrt{2} c n \xi+2 \sqrt{2} d n \xi), c_{2}=c_{5}=c_{6}=0, c_{1}=-33 / 8 \\
c_{3} & =-3 / 2, c_{7}=1 / 8 \\
F_{13}(\xi) & =(\sqrt{1 / 2} c n \xi \pm d n \xi) /\left(1 / 2+(\sqrt{1 / 2} c n \xi \pm d n \xi)+(\sqrt{1 / 2} c n \xi \pm d n \xi)^{2}\right) \\
c_{2} & =c_{5}=c_{6}=0, c_{1}=9 / 2, c_{3}=6, c_{7}=-1 / 2 \\
F_{14}(\xi) & =\rho\left(\xi, D_{1}, D_{2}\right), c_{1}=c_{2}=c_{5}=c_{6}=0, c_{3}=-12, c_{7}=D_{1} / 2
\end{aligned}
$$

where:

$$
\xi=x \exp \left(\int \alpha_{1}(s) d s\right)+\int \alpha_{2}(s) \exp \left(\int \alpha_{1}(s) d s\right) d s,
$$

$D_{1}, D_{2}$ and $D_{3}$ are arbitrary constants, $A i(\xi)$ and $B i(\xi)$ are the first and the second Airy functions and $p(\cdot, \cdot, \cdot)$ is the Weierstrass elliptic function. Therefore, the GVGE with the forcing term has the following solution:

$$
u_{i}=\exp \left(-\int \gamma(s) d s\right)\left(F_{i}(\xi)-\int \delta(s) \exp \left(\int \gamma(s) d s\right) d s\right),
$$

where $F_{i}(\xi)(i=1,2, \cdots 14)$ is determined by the above system.

Remark 3. Let $c_{1}=-6 a_{4}, c_{2}=c_{6}=0, c_{3}=-3 a_{3}, c_{5}=-a_{2}, c_{7}=-a_{1} / 2$. By solving Equation (34), one can obtain all solutions $\phi$ of Appendix A, Appendix B and Appendix C in [32].

Choosing some values for the given functions and parameters, we discuss the similarity solutions and the corresponding figures of the generalized variable-coefficient Gardner equation with the forcing 
term. Figure 1 is the plot of the one-solitary-wave solution, which can be obtained by choosing $a(s)=1, b(s)=2, h(s)=1, d(s)=f(s)=R(s)=0, C_{1}=0$. In this case, Equation (1) becomes a constant-coefficient Gardner equation, which can be reduced to the following third-order ordinary differential equation:

$$
F^{\prime \prime \prime}+2 F^{2} F^{\prime}+F F^{\prime}+c F^{\prime}=0,
$$

which has the solution $F_{7}(\xi), \xi=x+c s$ and $u=F(\xi)$ are similarity variables obtained by $\sigma_{4}=0$. By choosing $a(s)=12 e^{s}, b(s)=-6, h(s)=1, d(s)=f(s)=0$ and the forcing term $R(s)=e^{s}$, we find that Equation (1) has the solution $u(x, s)=\tanh \left(x+2 s-3 e^{2 s}\right)+e^{s}$, the plot of which is Figure 2 . There is a changing amplitude in the wave propagation for the effects of $a(s)=12 e^{s}$ and $R(s)=e^{s}$ in Figure 2.

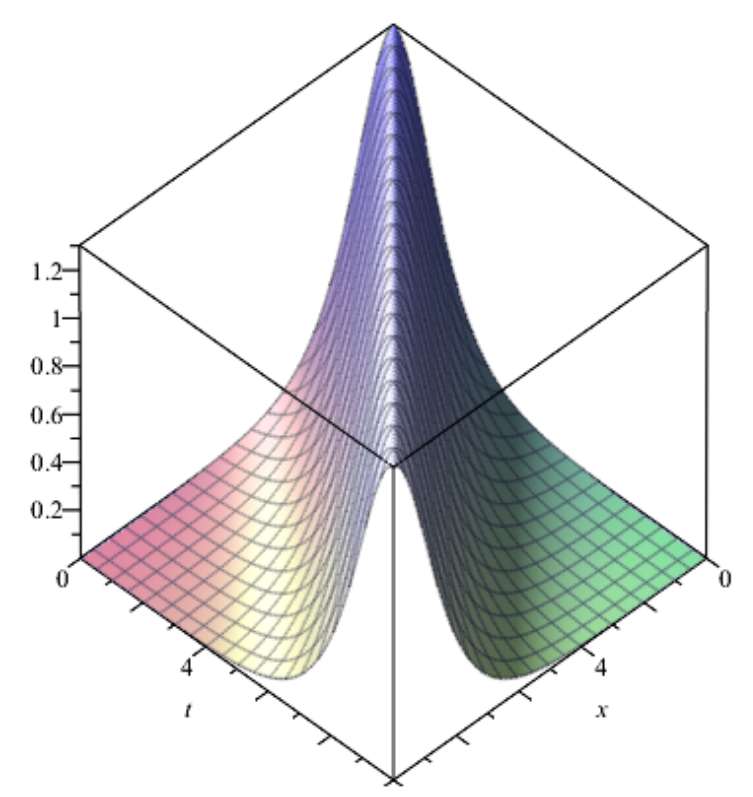

Figure 1. The solution of the constant-coefficient Gardner equation with $c=1$.

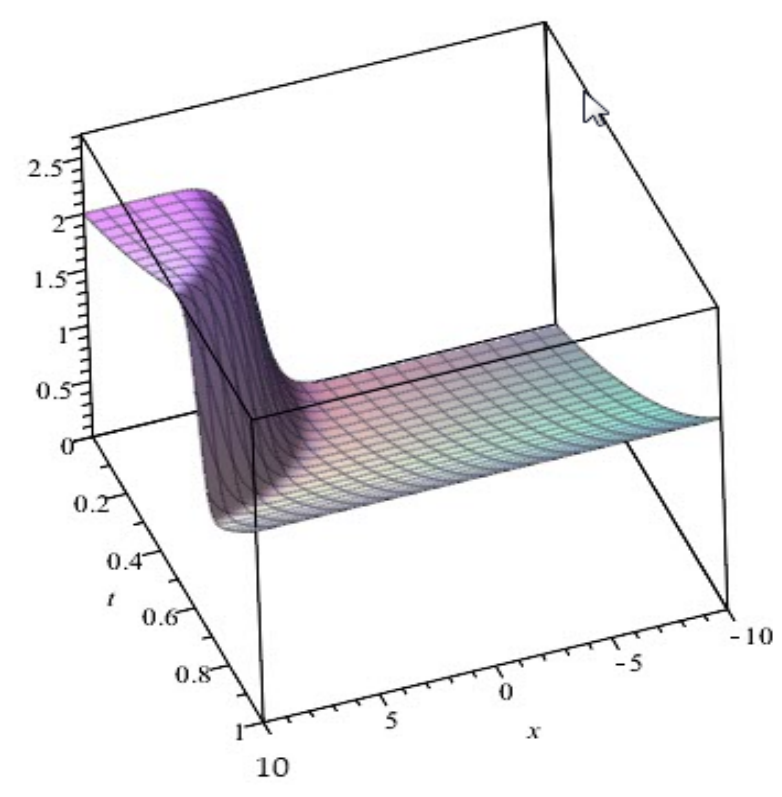

Figure 2. The solution of Equation (1) with $R(s)=e^{s}$. 


\section{Conclusions}

The compatibility method is a systematic and effective method for solving nonlinear evolution equations. Such a method can be used to obtain abundant symmetry reductions and similarity solutions. In addition, it is capable of greatly reducing the computational complexity in comparison to the non-classical group methods. In this paper, the method is first used to solve variable coefficients nonlinear evolution equations with a forcing term. With the aid of Maple, four types of symmetry of GVGE have been presented. Then, by solving the characteristic equations of the symmetry, a variety of similarity solutions has been obtained, which contain Airy function solutions $u_{2}$, triangular periodic solutions $u_{5}, u_{8}, u_{9}$, rational function solutions $u_{3}, u_{10}$, Weierstrass elliptic function solutions $u_{14}$, and so on. Many solutions found in this paper have not been reported in the literature.

Acknowledgments: This work was supported by the National Natural Science Foundation of China under Grants 61503002, 61304066 and 61573008.

Author Contributions: Jianping Zhou conceived of and designed the solving process of the generalized Gardner equation. Yuan Wang and Yang Wang contributed to the contents and writing of the manuscript. Zhiian Yan and Zhen Wang provided guidance and recommendations for this research. All authors have read and approved the final manuscript.

Conflicts of Interest: The authors declare no conflict of interest.

\section{References}

1. Lou, S.; Huang, G.; Ruan, H. Exact solitary waves in a convecting fluid. J. Phys. A Gen. Phys. 1991, 24, 587-590.

2. Wang, H.; Wang, Y.; Dong, H. Interaction solutions of a (2+1)-dimensional dispersive long wave system. Comput. Math. Appl. 2018, doi:10.1016/j.camwa.2017.12.032.

3. Gardner, C.S.; Greene, J.M.; Kruskal, M.D.; Miura, R.M. Method for solving the Korteweg-deVries equation. Phys. Rev. Lett. 1967, 19, 1095.

4. Wang, M.; Zhou, Y.; Li, Z. Applications of a homogeneous balance method to exact solutions of nonlinear equations in mathematical physics. Phys. Lett. A 1996, 216, 67-75.

5. Naher, H.; Abdullah, F.A. New approach of $\left(G^{\prime} / G\right)$-expansion method and new approach of generalized $\left(G^{\prime} / G\right)$-expansion method for nonlinear evolution equation. AIP Adv. 2013, 3, 032116.

6. Dong, H.; Zhao, K.; Yang, H.; Li, Y. Generalised (2+1)-dimensional super MKdV hierarchy for integrable systems in soliton theory. East Asian J. Appl. Math. 2015, 5, 256-272.

7. Wazwaz, A.M. A sine-cosine method for handling nonlinear wave equations. Math. Comput. Model. 2004, 40, 499-508.

8. Fang, Y.; Dong, H.; Hou, Y.; Kong, Y. Frobenius integrable decompositions of nonlinear evolution equations with modified term. Appl. Math. Comput. 2014, 226, 435-440.

9. Zhang, S.; Li, J.; Zhou, Y. Exact solutions of non-linear lattice equations by an improved exp-function method. Entropy 2015, 17, 3182-3193.

10. Demiray, S.T.; Bulut, H. New exact solutions of the new Hamiltonian amplitude-equation and Fokas Lenells equation. Entropy 2015, 17, 6025-6043.

11. Yang, X.; Gao, F.; Srivastava, H.M. Exact travelling wave solutions for the local fractional two-dimensional Burgers-type equations. Comput. Math. Appl. 2017, 73, 203-210.

12. Hirota, R. Exact solution of the Korteweg-de Vries equation for multiple collisions of solitons. Phys. Rev. Lett. 1971, 27, 1192-1194.

13. Zhao, H.; Ma, W. Mixed lump-kink solutions to the KP equation. Comput. Math. Appl. 2017, 74, $1399-1405$.

14. Ma, W.; Zhou, Y. Lump solutions to nonlinear partial differential equations via Hirota bilinear forms. J. Differ. Equ. 2018, 264, 2653-2659.

15. Zhao, Q.; Li, X.; Liu, F. Two integrable lattice hierarchies and their respective Darboux transformations. Appl. Math. Comput. 2013, 219, 2693-5705.

16. Xu, X.; Sun, Y. An integrable coupling hierarchy of Dirac integrable hierarchy, its Liouville integrability and Darboux transformation. J. Nonlinear Sci. Appl. 2017, 10, 3328-3343.

17. Bluman, G.W.; Cole, J.D. The general similarity of the heat equation. J. Math. Mech. 1969, 18, 1025-1042. 
18. Clarkson, P.A.; Kruskal, M.D. New similarity solutions of the Boussinesq equation. J. Math. Phys. 1989, 30, 2201-2213.

19. Cherniha, R.; King, J.R. Lie symmetries of nonlinear multidimensional reaction-diffusion systems: I. J. Phys. A Math. Gen. 2000, 33, 267.

20. Cherniha, R.; King, J.R. Lie symmetries of nonlinear multidimensional reaction-diffusion systems: II. J. Phys. A Math. Gen. 2003, 36, 405-425.

21. Moleleki, L.D.; Johnpillai, A.G.; Khalique, C.M. Symmetry reductions and exact solutions of a variable coefficient (2+1)-Zakharov-Kuznetsov equation. Math. Comput. Appl. 2012, 17, 132-139.

22. Nucci, M.C.; Sanchini, G. Symmetries, Lagrangians and conservation laws of an Easter Island population model. Symmetry 2015, 7, 1613-1632.

23. Dong, H.; Chen, T.; Chen, L.; Zhang, Y. A new integrable symplectic map and the lie point symmetry associated with nonlinear lattice equations. J. Nonlinear Sci. Appl. 2016, 9, 5107-5118.

24. Cherniha, R.; Davydovych, V.; Liliia, M. Lie symmetries of the shigesada-Kawasaki-Teramoto system. Commun. Nonlinear Sci. Numer. Simul. 2017, 45, 81-92.

25. Huang, D.; Li, X.; Yu, S. Lie symmetry classification of the generalized nonlinear Beam equation. Symmetry 2017, 9, 115.

26. Hydon, P.E. Symmetry Methods for Differential Equations: A Beginner's Guide; Cambridge University Press: New York, NY, USA, 2000.

27. Yan, Z.; Liu, X. Symmetry and similarity solutions of variable coefficient generalized Zakharov-Kuznetsov equation. Appl. Math. Comput. 2006, 180, 288-294.

28. Yan, Z.; Zhou, J.; Liu, X. Symmetry reductions and similarity solutions of the (3+1)-dimensional breaking soliton equation. Appl. Math. Comput. 2008, 201, 333-339.

29. Yan, Z.; Zhou, J. New explicit solutions of (1+1)-dimensional variable-coefficient Broer-Kaup system. Commun. Theor. Phys. 2010, 54, 965.

30. Zhang, S. Exact solutions of Wick-type stochastic Korteweg-de Vries equation. Can. J. Phys. 2012, 90, 181-186.

31. Gupta, R.; Singh, M. Nonclassical symmetries and similarity solutions of variable coefficient coupled KdV system using compatibility method. Nonlinear Dyn. 2017, 87, 1543-1552.

32. Hong, B.; Lu, D. New exact solutions for the generalized variable-coefficient Gardner equation with forcing term. Appl. Math. Comput. 2012, 219, 2732-2738.

33. Li, J.; Xu, T.; Meng, X.; Zhang, Y.; Zhang, H.; Tian, B. Lax pair: Bäcklund transformation and N-soliton-like solution for a variable-coefficient Gardner equation from nonlinear lattice, plasma physics and ocean dynamics with symbolic computation. J. Math. Anal. Appl. 2007, 336, 1443-1455.

34. Xu, X.; Meng, X.; Gao, Y.; Wen, X. Analytic N-solitary-wave solution of a variable-coefficient Gardner equation from fluid dynamics and plasma physics. Appl. Math. Comput. 2009, 210, 313-320.

35. Bulut, H.; Sulaiman, T.; Baskonus, H.M.; Sandulyak, A.A. New solitary and optical wave structures to the (1+1)-dimensional combined KdV-mKdV equation. Optik 2017, 135, 327-336.

36. Latif, M.A. Some exact solutions of $\mathrm{KdV}$ equation with variable coefficient. Commun. Nonlinear Sci. Numer. Simul. 2009, 16, 1783-1786.

37. Johnpillai, A.G.; Khalique, C.M. Group analysis of $\mathrm{KdV}$ equation with time dependent coefficients. Appl. Math. Comput. 2010, 216, 3761-3771.

38. Johnpillai, A.G.; Khalique, C.M. Lie group classification and invariant solutions of mKdV equation with time-dependent coefficients. Commun. Nonlinear Sci. Numer. Simul. 2011, 16, 1207-1215.

(c) 2018 by the authors. Licensee MDPI, Basel, Switzerland. This article is an open access article distributed under the terms and conditions of the Creative Commons Attribution (CC BY) license (http://creativecommons.org/licenses/by/4.0/). 